\title{
Surface plasmon resonance spectroscopy as an alternative for sensing heavy metal ions : a review
}

\begin{abstract}
Purpose - The purpose of this paper is to review the novel application of surface plasmon resonance (SPR) in sensing heavy metal ions and the development of SPR to become an alternative heavy metal ions sensor. Design/methodology/ approach - The possible dangerous toxic effects of heavy metal ions are revealed in the short introduction. The existing conventional methods for sensing heavy metal ions and their drawbacks are also discussed. To overcome these drawbacks, SPR has been investigated from the basic principle to the potential alternative in sensing heavy metal ions. Findings - Application of SPR in sensing heavy metal ions emerged a decade ago. A wide range of active layers or recognition elements (e.g. polymer, protein, nanoparticles) have been developed to combine with SPR. The detection limit, sensitivity and selectivity of SPR sensing in heavy metal ions have been improved from time to time, until the present. Originality/value - This paper provides up-todate and systematic information on SPR sensing for heavy metal ions. Different advancements on active layers or recognition molecules have been discussed in detail and arranged in the order of their chronological evolution. The present review may provide researchers with valuable information regarding novel heavy metal ions sensor using SPR and encourage them to take this area for further research and development.
\end{abstract}

Keyword: Active layers; Heavy metal ions; Heavy metals; Recognition elements; Sensors; Surface plasmon resonance. 